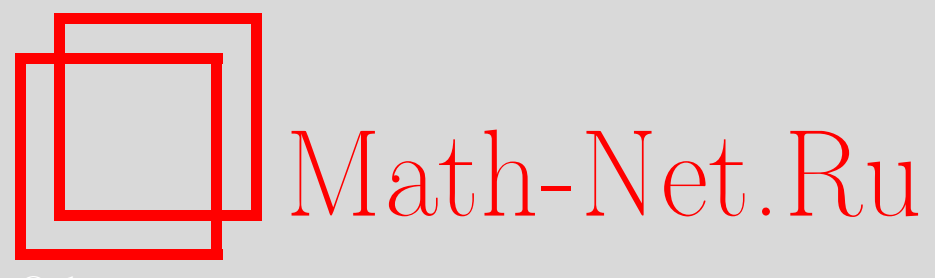

А. Канунников, И. Воробьев, Линейная независимость радикалов, Квант, 2021, номер 3, 12-20

DOI: https://doi.org/10.4213/kvant20210302

Использование Общероссийского математического портала Math-Net.Ru подразумевает, что вы прочитали и согласны с пользовательским соглашением http://www.mathnet.ru/rus/agreement

Параметры загрузки:

IP : 54.224 .135 .184

26 апреля 2023 г., $14: 42: 45$

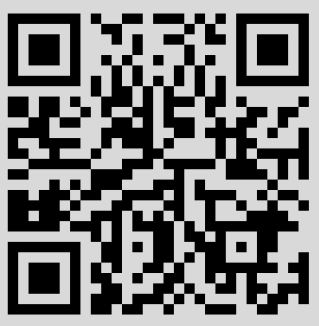




\section{Линейная независимость \\ радикалов}

\section{А.КАНУННИКОВ, И.ВОРОБЬЕВ}

\section{§1. Введение}

Как доказать иррациональность чисел

$\sqrt[3]{\frac{121}{111}}, \sqrt{2}+\sqrt{3}, \sqrt{2}+\sqrt[3]{3}, \sqrt{2}+\sqrt{3}+\sqrt{5}+\sqrt{7}$,

$$
\sqrt[5]{3}-\sqrt[5]{2}, \sqrt{\frac{3}{5}}+\frac{\sqrt[17]{2021 !}}{2021}+5^{5^{5}} \sqrt{\frac{7^{7^{7}}}{3^{3^{3}}}} ?
$$

Иррациональность одного радикала простой, чисто арифметический вопрос, сводящийся к разложению на простые множители.

Лемма 1. ${ }^{1}$ Пусть $A, B, k \in \mathbb{N} u$ НОД $(A, B)=$ = 1. тогда $\sqrt[k]{A / B} \in \mathbb{Q}$, если и только если показатели степеней всех простых делителей в разложениях чисел А и В кратны $k$.

$$
\text { Число } \sqrt[3]{\frac{121}{111}}=\sqrt[3]{\frac{11^{2}}{3 \cdot 37}} \text { иррационально по }
$$

лемме 1. Если $\sqrt{2}+\sqrt{3} \in \mathbb{Q}$, то $(\sqrt{2}+\sqrt{3})^{2}=$ $=5+2 \sqrt{6} \in \mathbb{Q}$, откуда $\sqrt{6} \in \mathbb{Q}$, что неверно по той же лемме. Предположив, что $\sqrt{2}+\sqrt[3]{3}=a \in \mathbb{Q}$, возведем равенство $\sqrt[3]{3}=$ $=a-\sqrt{2}$ в куб: $3=a^{3}+6 a-\left(3 a^{2}+2\right) \sqrt{2}$ и придем к противоречию: $\sqrt{2}=\frac{a^{3}+6 a-3}{3 a^{2}+2} \in$ $\in \mathbb{Q}$. Однако остальные числа «голыми руками не возьмешь»: незатейливое возведение в степень только приумножит количество радикалов.

«Неужели, прочитав статью, я пойму, почему даже последнее, нарочито дикое,

\footnotetext{
1 Леммы и теоремы мы предлагаем читателю доказывать самостоятельно, так же как и решать задачи. Доказательства и решения будут приведены в следующем номере журнала.
}

DOI: https://doi.org/10.4213/kvant20210302 число иррационально?»- наверное, думает читатель в эту минуту. «Да, - ответим мы, - если только вы умеете делить многочлены с остатком и извлекать корни из комплексных чисел. Если пока не умеете, то во всяком случае вы разберетесь со сколь угодно длинными суммами квадратных радикалов».

На самом деле мы докажем даже большее: между корнями из рациональных чисел не существует линейных соотношений с рациональными коэффициентами, кроме очевидных отношений пропорциональности, например $\sqrt{8}=2 \sqrt{2}$. Вот точная формулировка.

Теорема 1. Пусть $N, k_{1}, \ldots, k_{N} \in \mathbb{N}, N>1$, $0<Q_{1}, \ldots, Q_{N} \in \mathbb{Q} \quad$ u $\sqrt[k_{i}]{Q_{i}} / \sqrt[k_{j}]{Q_{j}} \notin \mathbb{Q} \quad$ nри $i \neq j$. Тогда равенство

$a_{1} \sqrt[k_{1}]{Q_{1}}+\ldots+a_{N} \sqrt[k_{N}]{Q_{N}}=0$,

где $a_{1}, \ldots, a_{N} \in \mathbb{Q}$,

выполняется только при $a_{1}=\ldots=a_{N}=0$.

В частности, при $Q_{1}=1$ получим, что сумма $\sqrt[k_{2}]{Q_{2}}+\ldots+\sqrt[k_{N}]{Q_{N}}$ иррациональна, так как равенство $a_{1} \sqrt[k_{1}]{1}+\sqrt[k_{2}]{Q_{2}}+\ldots$ $\ldots+\sqrt[k_{N}]{Q_{N}}=0$ не может выполняться ни при каком $a_{1} \in \mathbb{Q}$.

Чтобы применять теорему 1, нужно проверять иррациональность отдельных радикалов $\sqrt[k_{i} k_{j}]{Q_{i}^{k_{j}} / Q_{j}^{k_{i}}}$ по лемме 1.

Задача 1. Выведите из теоремы 1 и леммы 1 иррачиональность трех последних чисел в начале статьи.

На языке векторов утверждение теоремы 1 формулируется так: числа $\sqrt[k_{1}]{Q_{1}}, \ldots, \sqrt[k_{N}]{Q_{N}}$ линейно независимь над $\mathbb{Q}$ (сравните с 
некомпланарными векторами на рисунке 1). Условие $\sqrt[k_{i}]{Q_{i}} / \sqrt[k_{j}]{Q_{j}} \notin \mathbb{Q}$ $\mathrm{B}$ этих терминах озна-

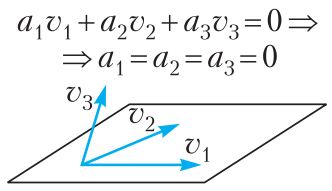

РИс. 1 чает линейную независимость чисел $\sqrt[k_{i}]{Q_{i}}$ и $\sqrt[k_{j}]{Q_{j}}$ над $\mathbb{Q}$ (линейная независимость двух векторов - это просто их неколлинеарность).

Вообще, взгляд на алгебраические числа (в частности, на радикалы) как на векторы оказывается естественным и продуктивным - он позволяет применять геометрические идеи к алгебраическим задачам (см. [4]).

Сформулируем более удобную для доказательства, но, как окажется, равносильную теорему.

Tеорема 2. Пусть $k, n \in \mathbb{N}, p_{1}, \ldots, p_{n}-$ pa3- $^{-}$ ные простые числа, $r_{1}=\sqrt[k]{p_{1}}, \ldots, r_{n}=\sqrt[k]{p_{n}}$. Тогда система $\left\{r_{1}^{l_{1}} \ldots r_{n}^{l_{n}} \mid 0 \leq l_{1}, \ldots, l_{n}<k\right\}$ из $k^{n}$ чисел линейно независима над $\mathbb{Q}$.

Эту систему удобно представлять в виде $n$-мерной решетки (рис. 2).
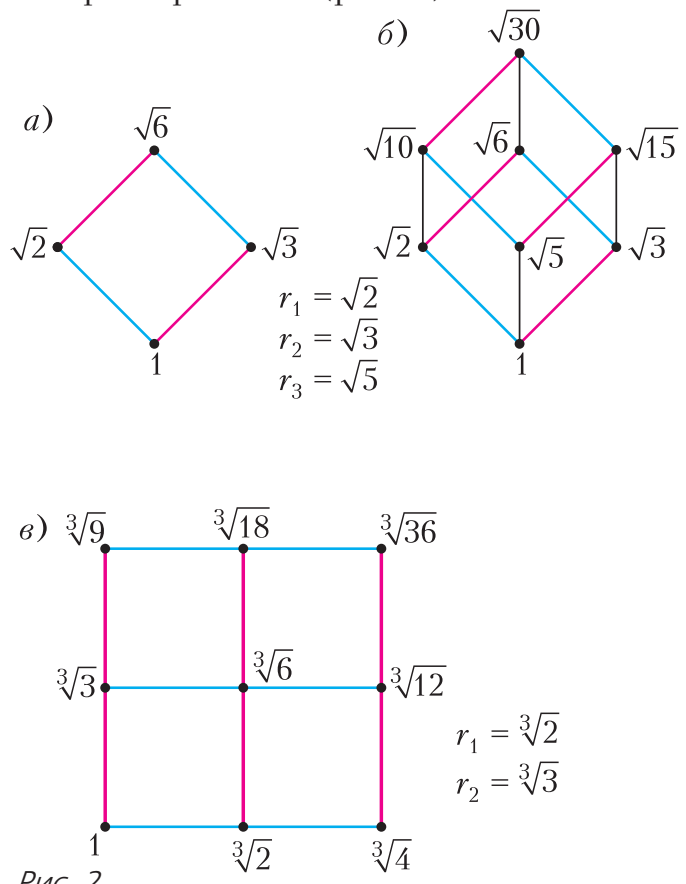

Pnc. 2
Задача 2. а) Как связаны наборы чисел на параллельных сторонах квадрата на рисунке 2, а и на параллельных ребрах и гранях куба на рисунке 2,б? б) Попробуйте нарисовать гиперкуб (4-мерньй куб) и расставить в его вериинах радикаль по тому же принщипу.

Задача 3. Выведите теоремы 1 и 2 друг из друга.

Указание. $1 \Rightarrow 2$ : числа из системы в теореме 2 попарно непропорииональны над $\mathbb{Q} ; 2 \Rightarrow 1$ : обобщите рассуждение из следующего примера.

Пример 1. Сведем линейную независимость (по умолчанию - над $\mathbb{Q}$ ) чисел 1 , $\sqrt{1 / 2}, \sqrt[3]{4 / 3}$ к теореме 2. Выравняем показатели корней: $1, \sqrt[6]{1 / 2^{3}}, \sqrt[6]{2^{4} / 3^{2}}$, домножим второе число на 2 , а третье - на $3: 1, \sqrt[6]{2^{3}}$, $\sqrt[6]{2^{4} \cdot 3^{4}}$ (это не влияет на линейную независимость). Получим подсистему системы $\left\{\sqrt[6]{2} \sqrt[l_{1}]{3^{l_{2}}} \mid 0 \leq l_{1}, l_{2} \leq 5\right\}$, а она линейно независима по теореме 2.

В литературе для школьников неоднократно обсуждался случай квадратных радикалов (см. [2, 4, 6]). В §2 мы разберем его на примерах, подводя ко многим, пусть и не всем, идеям в общей ситуации. Теорема 2 при $n=1$ доказана в [5]. Общий случай разобран, например, в научной статье [7] с помощью теории Галуа. В §3 мы докажем теорему 2 достаточно элементарно, попутно сообщая необходимые сведения об алгебраических числах, интересные и сами по себе. В конце мы приведем совсем короткое доказательство теоремы 1, доступное первокурснику. Все необходимое для понимания собрано в §4 (в следующем номере журнала).

\section{§2. Случай квадратных радикалов}

Теорему 2 при $k=2$ можно доказать индукцией по $n$. База $n=1$ : линейная независимость 1 и $\sqrt{p_{1}}$ означает просто иррациональность $\sqrt{p_{1}}$ и имеет место по лемме 1. Разберем случаи $n=2,3$ на конкретных примерах - так проще объяснить и 
понять идеи доказательства. Полное рассуждение с любым $k$ индукцией по $n$ проведем в $\$ 3$.

Пример 2. Докажем, что числа $1, \sqrt{2}$, $\sqrt{3}, \sqrt{6}$ (см. рис. $2, a$ ) линейно независимы. Пусть $a+b \sqrt{2}+c \sqrt{3}+d \sqrt{6}=0$, где $a, b, c, d \in$ $\in \mathbb{Q}$. Выделим $\sqrt{3}: a+b \sqrt{2}+(c+d \sqrt{2}) \sqrt{3}=$ $=0$. Если $c+d \sqrt{2}=0$, то и $a+b \sqrt{2}=0$, а тогда, ввиду иррациональности $\sqrt{2}$, $c=d=0$ и $a=b=0$. Если же $c+d \sqrt{2} \neq 0$, TO

$$
\begin{aligned}
& \sqrt{3}=- \frac{a+b \sqrt{2}}{c+d \sqrt{2}}= \\
&=-\frac{(a+b \sqrt{2})(c-d \sqrt{2})}{c^{2}-2 d^{2}}=A+B \sqrt{2},
\end{aligned}
$$

где $A=\frac{2 b d-a c}{c^{2}-2 d^{2}}, B=\frac{a d-b c}{c^{2}-2 d^{2}} \in \mathbb{Q}$.

Возведем равенство $\sqrt{3}=A+B \sqrt{2}$ в квадрат:

$3=A^{2}+2 B^{2}+2 A B \sqrt{2} \Rightarrow A B=0$,

$$
\text { иначе } \sqrt{2} \in \mathbb{Q} \text {. }
$$

При $A=0$ получаем $\sqrt{3 / 2}=B \in \mathbb{Q}$, при $B=0$ получаем $\sqrt{3}=A \in \mathbb{Q}-$ противоречие с леммой 1.

Мы свели линейную независимость чисел на рисунке $2, a$ к «инородности» $\sqrt{3}$ по отношению к множеству

$$
\mathbb{Q}+\mathbb{Q} \sqrt{2}=\{a+b \sqrt{2} \mid a, b \in \mathbb{Q}\}
$$

(формально, $\sqrt{3}$ не лежит в нем), подобно тому как $\sqrt{2}$ инородно по отношению к $\mathbb{Q}$. При этом оказалось важно, что в множестве $\mathbb{Q}+\mathbb{Q} \sqrt{2}$ можно не только складывать, вычитать, умножать, но и делить (не на 0), как и в $\mathbb{Q}$. Прежде чем сделать следующий шаг, введем несколько понятий.

Немного теории. Множество чисел, содержащее 0 и 1 и замкнутое относительно четырех арифметических действий, называется числовым полем. Слово «числовое» мы будем опускать. Итак, $K$ - поле, если $0,1 \in K \quad$ и для любых $a, b \in K \quad$ верно $a \pm b, a b \in K$ и $a / b \in K$ при $b \neq 0$. Легко понять, что $\mathbb{Q}-$ поле, причем «самое маленькое» - любое поле его содержит. ${ }^{2}$ Если поле $K$ содержится в поле $L$, то говорят, что $K-$ nодполе в $L$. В этом параграфе все поля лежат в поле $\mathbb{R}$ действительных чисел.

Числа $x_{1}, \ldots, x_{n}$ называются линейно независимыми над полем $K$, если равенство $a_{1} x_{1}+\ldots+a_{n} x_{n}=0$, где $a_{1}, \ldots, a_{n} \in K$, выполняется только при $a_{1}=\ldots=a_{n}=0$. Например, числа 1 и $\sqrt{2}$ линейно независимы над $\mathbb{Q}$, но линейно зависимы над $\mathbb{R}$ (равенство $a_{1} \cdot 1+a_{2} \sqrt{2}=0$ верно при $a_{1}=\sqrt{2}$ и $\left.a_{2}=-1\right)$.

Лемма 2 (свойства линейной зависимости над произвольным полем $K$ ).

1) Система, содержащая 0 или два числа, отношение которых лежит в $\mathrm{K}$, линейно зависима над K.

2) Подсистема линейно независимой системы линейно независима.

3) Система 1, х линейно независима над $K$, если и только если $x \notin K$.

4) Коэффичиенты $a_{1}, \ldots, a_{n} \in K$ в записи числа $a_{1} x_{1}+\ldots+a_{n} x_{n}$ определены однозначно, если и только если система $x_{1}, \ldots, x_{n}$ линейно независима над $K$.

Наименьшее (по включению) поле, содержащее поле $K$ и числа $\alpha_{1}, \ldots, \alpha_{n}$, обозначается $K\left(\alpha_{1}, \ldots, \alpha_{n}\right)$ и называется полем, порожденным над $K$ этими числами. Опишем поле, порожденное над $K$ одним квадратным радикалом $\sqrt{d} \notin K$, где $d \in K$. Поле $K(\sqrt{d})$, очевидно, состоит из отношений чисел вида $a+b \sqrt{d}$, где $a, b \in K$. Но от иррациональности в знаменателе можно избавиться, домножив на сопряженное $a-b \sqrt{d}$ :

$$
\frac{1}{a+b \sqrt{d}}=\frac{a-b \sqrt{d}}{a^{2}-d b^{2}}
$$

(как в примере 2). Значит,

$$
K(\sqrt{d})=\{a+b \sqrt{d} \mid a, b \in K\} .
$$

\footnotetext{
2 Бывают и другие поля: поле вычетов $\mathbb{Z}_{p}$, поле рациональных функций и др. Любое поле содержит либо $\mathbb{Q}$, либо $\mathbb{Z}_{p}$.
} 
Пример 3. Пусть $p, q$ - различные простые числа. Аналогично примеру 2, $\sqrt{q} \notin \mathbb{Q}(\sqrt{p})$ и числа $1, \sqrt{p}, \sqrt{q}, \sqrt{p q}$ линейно независимы над $\mathbb{Q}$. Поэтому

$$
\begin{aligned}
& \mathbb{Q}(\sqrt{p}, \sqrt{q})=\mathbb{Q}(\sqrt{p})(\sqrt{q})= \\
& \quad=\{a+b \sqrt{p}+c \sqrt{q}+d \sqrt{p q} \mid a, b, c, d \in \mathbb{Q}\},
\end{aligned}
$$

причем запись чисел в таком виде однозначна.

Пример 4. Докажем, что числа на рисунке $2, б$ линейно независимы над $\mathbb{Q}$. Пусть $a_{1}+a_{2} \sqrt{2}+\ldots+a_{8} \sqrt{30}=0$, где $a_{1}, a_{2}, \ldots, a_{8} \in \mathbb{Q}$.

Шаг 1. Вынося $\sqrt{5}$ за скобки, получим равенство вида $A+B \sqrt{5}=0$, где $A, B \in$ $\in \mathbb{Q}(\sqrt{2}, \sqrt{3}):$

$$
\begin{aligned}
& \underbrace{a_{1}+a_{2} \sqrt{2}+a_{3} \sqrt{3}+a_{5} \sqrt{6}}_{A}+ \\
& \quad+\underbrace{\left(a_{4}+a_{6} \sqrt{2}+a_{7} \sqrt{3}+a_{8} \sqrt{6}\right)}_{B} \sqrt{5}=0 .
\end{aligned}
$$

Достаточно доказать, что $A=B=0$. Тогда, согласно примеру 2, $a_{1}=a_{2}=a_{3}=a_{5}=$ $=0$ и $a_{4}=a_{6}=a_{7}=a_{8}=0$.

Шаг 2. Надо доказать, что числа 1 и $\sqrt{5}$ линейно независимы над полем $\mathbb{Q}(\sqrt{2}, \sqrt{3})$. Это равносильно условию $\sqrt{5} \notin \mathbb{Q}(\sqrt{2}, \sqrt{3})$ (пункт 3 леммы 2).

Шаг 3. Предположим, что $\sqrt{5} \in \mathbb{Q}(\sqrt{2}, \sqrt{3})$, т.е., с учетом описания этого поля в примеpe 3 ,

$\sqrt{5}=a+b \sqrt{2}+c \sqrt{3}+d \sqrt{6}$,

$$
\text { где } a, b, c, d \in \mathbb{Q} \text {. }
$$

Большое число радикалов может отбить желание возводить в квадрат. Однако это можно сделать по-умному, предварительно сгруппировав слагаемые одним из двух способов:

$$
\begin{aligned}
& \underbrace{a+b \sqrt{2}}_{C}+\underbrace{(c+d \sqrt{2})}_{D} \sqrt{3}=\sqrt{5}= \\
& =\underbrace{a+b \sqrt{3}}_{C^{\prime}}+\underbrace{(b+d \sqrt{3})}_{D^{\prime}} \sqrt{2},
\end{aligned}
$$

и «не тревожить» $C, D, C^{\prime}, D^{\prime}$. Возведем в квадрат первое равенство:

$$
C^{2}+3 D^{2}+2 C D \sqrt{3}=5 .
$$

Так как числа 1 и $\sqrt{3}$ линейно независимы над полем $\mathbb{Q}(\sqrt{2})$, то $C D=0$. Аналогично, $C^{\prime} D^{\prime}=0$. Всего 4 варианта: $C=C^{\prime}=0$, $C=D^{\prime}=0, C^{\prime}=D=0$ или $D=D^{\prime}=0$. Если $C=C^{\prime}=0$, то $a=b=c=0, \quad$ поэтому $\sqrt{5}=d \sqrt{6}$, а это противоречит лемме $1 . \mathrm{B}$ других случаях получается аналогичное противоречие, так как в правой части равенства (2) остается лишь одно ненулевое слагаемое.

Задача 4. Докажите теорему 2 при $k=2$ : сделайте шаг индукции от $n-1 \kappa n$, следуя примеру 4.

Прежде чем переходить к радикалам более высокой степени, обсудим, как можно было сделать последний шаг с прицелом на общий случай - без возведения в квадрат. Согласитесь, даже столь безобидное равенство, как $\sqrt[5]{3}=a+b \sqrt[5]{2}(a, b \in \mathbb{Q})$, едва ли удастся привести к противоречию лобовым возведением в пятую степень... Что если в равенствах (3) перейти к сопряженным числам?

Назовем числа вида $a \pm b \sqrt{d}$ над полем $K$, где $a, b, d \in K, \sqrt{d} \notin K$, сопряженными. В равенствах (3) сопряженным к числу $C+D \sqrt{3}$ над полем $\mathbb{Q}(\sqrt{2})$ будет $C-D \sqrt{3}$, а сопряженным к числу $C^{\prime}+D^{\prime} \sqrt{2}$ над полем $\mathbb{Q}(\sqrt{3})$ будет $C^{\prime}-D^{\prime} \sqrt{2}$. В то же время сопряженным к числу $\sqrt{5}$ над обоими полями будет $-\sqrt{5}$. Раз числа равны, то равны и их сопряженные:

$$
C-D \sqrt{3}=-\sqrt{5}=C^{\prime}-D^{\prime} \sqrt{2} .
$$

Отсюда и из (3) получаем $C=C^{\prime}=0$, что невозможно, как показано выше.

Увы, несмотря на кажущуюся строгость, это рассуждение содержит «дыру», однако его можно спасти. Логическая ошибка довольно тонкая, и читатель, который ее обнаружит и исправит, вправе собой гордиться.

Задача 5. Почему переход от (3) $к$ (4) не обоснован и как его доказать? 
Переход к сопряженным часто бывает эффективен. Вот несколько олимпиадных задач на эту тему.

Задача 6. Существуют ли такие рациональные числа $a, b, c, d$, что $(a+b \sqrt{2})^{2}+$ $+(c+d \sqrt{2})^{2}=7+5 \sqrt{2} ?$

Задача 7. Найдите первые 1000 знаков после запятой в десятичной записи числа $(6+\sqrt{35})^{1000}$.

Задача 8. Докажите, что произведение $2^{100}$ чисел $\pm \sqrt{1} \pm \sqrt{2} \pm \ldots \pm \sqrt{99} \pm \sqrt{100}-$ полный квадрат.

\section{§3. Общий случай}

Начнем доказывать теорему 2 при произвольном $k$, следуя плану из примера 4. При этом на каждом шаге будут возникать новые трудности. Чем дальше в лес - тем больше дров.

Шаг 1: отщепление последнего радикала. Линейное соотношение над $\mathbb{Q}$ (т.е. с коэффициентами из $\mathbb{Q}$ ) девяти чисел на рисунке 2,в можно записать в виде

$$
f_{0}(\sqrt[3]{2})+f_{1}(\sqrt[3]{2}) \sqrt[3]{3}+f_{2}(\sqrt[3]{2}) \sqrt[3]{9}=0
$$

где $f_{0}, f_{1}, f_{2}$ - многочлены степени меньше 3 над $\mathbb{Q}$ (сгруппировали слагаемые, как в (1)). Поэтому линейная независимость этих чисел будет следовать из двух фактов:

числа $1, \sqrt[3]{3}, \sqrt[3]{9}$ линейно независимы над полем $\mathbb{Q}(\sqrt[3]{2}) ;(5)$

числа $1, \sqrt[3]{2}, \sqrt[3]{4}$ линейно независимы над полем $\mathbb{Q}$. (6)

В самом деле, из (5) получаем $f_{0}(\sqrt[3]{2})=$ $=f_{1}(\sqrt[3]{2})=f_{2}(\sqrt[3]{2})=0$, а тогда из (6) коэффициенты многочленов $f_{0}, f_{1}, f_{2}$ - нулевые. Это рассуждение несложно обобщить и свести теорему 2 к следующей.

Теорема 2'. В обозначениях теоремы 2 числа $1, r_{n}, \ldots, r_{n}^{k-1}$ линейно независимы над полем $\mathbb{Q}\left(r_{1}, \ldots, r_{n-1}\right)$.

Шаг 2: «инородность» последнего радикала. Пусть $K$ - любое поле, $r$ - такое число, что $r^{k} \in K$. Выясним, когда числа $1, r, \ldots, r^{k-1}$ линейно независимы над $K .(7)$

Иными словами, $r$ не должно быть корнем многочлена степени меньше $k$ с коэффициентами из $K$. При $k=2$ это просто означает, что $r \notin K$. При $k>2$ все гораздо сложнее и интереснее.

Немного теории. Множество многочленов над полем $K$ обозначается $K[x]$. Многочлен над $K$ положительной степени, который не раскладывается в произведение многочленов над $K$ меньших степеней, называется неприводимым над $K$. Многочлены над полем можно делить с остатком («уголком»).

Пусть число $\alpha$ является корнем ненулевого многочлена над полем $K$, тогда оно называется алгебраическим над К. Среди всех таких многочленов только один имеет наименьшую степень и старший коэффициент 1 (если бы их было два, то их разность была бы многочленом меньшей степени с корнем $\alpha$ ). Он называется минимальным многочленом числа $\alpha$ над $K$, и мы будем его обозначать $\mu_{\alpha}^{K}(x)$ или $\mu_{\alpha}(x)$, если ясно, о каком поле $K$ идет речь.

Теорема 3. Многочлен $\mu_{\alpha}^{K}(x)=\mu_{\alpha}(x)$ обладает следующими свойствами:

1) его степень есть наименьшее такое $m \in \mathbb{N}$, что числа $1, \alpha, \ldots, \alpha^{m}$ линейно зави симы над $\mathrm{K}$;

2) многочлен $\mu_{\alpha}(x)$ неприводим над $K$;

3) любой многочлен из $K[x]$ с корнем $\alpha$ делится на $\mu_{\alpha}(x)$;

4) неприводимый над $K$ многочлен $p(x)$ с корнем $\alpha$ и старшим коэффичиентом 1 равен $\mu_{\alpha}(x)$.

Таким образом, (7) $\Leftrightarrow \mu_{r}^{K}(x)=x^{k}-r^{k} \Leftrightarrow$ $\Leftrightarrow$ двучлен $x^{k}-r^{k}$ неприводим над $K$. Например, утверждения (5) и (6) равносильны соответственно неприводимости двучлена $x^{3}-3$ над $\mathbb{Q}(\sqrt[3]{2})$ и двучлена $x^{3}-2$ над $\mathbb{Q}$, а это значит, что $\sqrt[3]{3} \notin \mathbb{Q}(\sqrt[3]{2})$ и $\sqrt[3]{2} \notin \mathbb{Q}$ (кубический многочлен неприводим над полем, если не имеет в нем корней). В общем случае условие «инородности» $r \notin K$ необходимо, но не достаточно. Хотя в нашем случае действующие лица $K$ 
и $r$ лежат в поле $\mathbb{R}$, мы выйдем в комплексную плоскость - поле $\mathbb{C}$, где двучлен $x^{k}-r^{k}$ раскладывается на линейные множители. По формуле Муавра [3]

$$
\begin{gathered}
x^{k}-r^{k}=(x-r)(x-r \varepsilon) \ldots\left(x-r \varepsilon^{k-1}\right), \\
\text { где } \varepsilon=\varepsilon_{k}=\cos \frac{2 \pi}{k}+i \sin \frac{2 \pi}{k}
\end{gathered}
$$

\section{(рис. 3).}

Лемма 3. Пусть $K-$ подполе в $\mathbb{R}, r \in \mathbb{R}$,

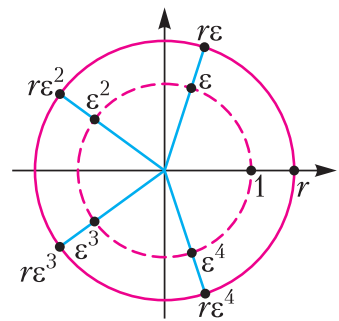

Pис. 3

$r^{k} \in K \quad$ u $\quad r, \ldots, r^{k-1} \notin$ К. Тогда двучлен $x^{k}-r^{k}$ неприводим над $K$.

(Для $r \notin \mathbb{R}$ лемма неверна; например, при $k=3$ и $r=\varepsilon$ имеем $\varepsilon, \varepsilon^{2} \notin \mathbb{R}$, но двучлен $x^{3}-1$ приводим.)

Пример 5. Для любого простого $p$ и натурального $k>1$ имеем $\sqrt[k]{p}, \ldots, \sqrt[k]{p^{k-1}} \notin \mathbb{Q}$ (лемма 1), поэтому двучлен $x^{k}-p$ неприводим над $\mathbb{Q}$ (лемма 3$)^{3}$, значит, $\mu_{\sqrt[k]{p}}^{\mathbb{Q}}(x)=x^{k}-p$.

Вообще, теорема 2' посредством леммы 3 сведена к теореме 2": в тех же обозначениях

$$
r_{n}, \ldots, r_{n}^{k-1} \notin \mathbb{Q}\left(r_{1}, \ldots, r_{n-1}\right) .
$$

При $n=1$ это верно по лемме 1. Чтобы прийти к противоречию при $n>1$, научимся записывать числа из $\mathbb{Q}\left(r_{1}, \ldots, r_{n-1}\right)$ в виде многочленов от $r_{1}, \ldots, r_{n-1}$. Например, условие $\sqrt[3]{3} \notin \mathbb{Q}(\sqrt[3]{2})$ запишется в виде $\sqrt[3]{3} \neq a+$ $+b \sqrt[3]{2}+c \sqrt[3]{4}$ при $a, b, c \in \mathbb{Q}$.

3 В [5] линейная независимость чисел $1, \sqrt[k]{p}, \ldots, \sqrt[k]{p^{k-1}}$ также сведена к неприводимости двучлена $x^{k}-p$, установленной по признаку Эйзенштейна.
Шаг 3: избавление от иррачиональности в знаменателе. Пресловутое «домножение на сопряженное» работает лишь с квадратными радикалами. Как действовать при $k>2$, сначала покажем на примере.

Пример 6. Избавимся от иррациональности в знаменателе дроби $\frac{1}{\sqrt[3]{4}+\sqrt[3]{2}+3}$. Обозначим $r=\sqrt[3]{2}$ и $f(x)=x^{2}+x+3$. Надо найти такой многочлен $u \in \mathbb{Q}[x]$, что $\frac{1}{f(r)}=u(r)$. Это значит, что многочлен $f(x) u(x)-1$ имеет корень $r$, а тогда делится на $\mu_{r}^{\mathbb{Q}}(x)=x^{3}-2$ (пример 5). Таким образом,

$$
u(x) f(x)+v(x)\left(x^{3}-2\right)=1
$$

для некоторого многочлена $v \in \mathbb{Q}[x]$. Многочлены $u$ и $v$ найдем с помощью алгоритма Евклида.

Алгоритм Евклида:

$$
\begin{aligned}
x^{3}-2 & =\left(x^{2}+x+3\right)(x-1)-2 x+1 ; \\
x^{2}+x+3 & =(2 x-1)\left(\frac{1}{2} x+\frac{3}{4}\right)+\frac{15}{4} .
\end{aligned}
$$

Обратный ход алгоритма Евклида:

$$
\begin{aligned}
\frac{15}{4} & =f(x)-\left(f(x)(x-1)-\left(x^{3}-2\right)\right)\left(\frac{1}{2} x+\frac{3}{4}\right)= \\
& =\left(x^{3}-2\right)\left(\frac{1}{2} x+\frac{3}{4}\right)+f(x)\left(-\frac{1}{2} x^{2}-\frac{1}{4} x+\frac{7}{4}\right) .
\end{aligned}
$$

Итак,

$\left(2 x^{2}+x-7\right)\left(x^{2}+x+3\right)-(2 x+3)\left(x^{3}-2\right)=-15$, откуда при $x=r$ получаем

$$
\frac{1}{\sqrt[3]{4}+\sqrt[3]{2}+3}=\frac{7-\sqrt[3]{2}-2 \sqrt[3]{4}}{15}
$$

Лемма 4 (об избавлении от иррациональности в знаменателе). Если число $\alpha$ алгебраично над полем $K u \operatorname{deg} \mu_{\alpha}(x)=k$, то каждое число в поле $K(\alpha)$ однозначно записывается в виде

$c_{0}+c_{1} \alpha+\ldots+c_{k-1} \alpha^{k-1}$, əде $c_{0}, c_{1}, \ldots, c_{k-1} \in K .(10)$ 
Задача 9. Избавьтесь от иррачиональности в знаменателях: а) $\frac{1}{1+\sqrt{2}-\sqrt{3}+\sqrt{6}}$; б) $\frac{1}{\sqrt[4]{27}+2 \sqrt[4]{3}-1}$.

Вернемся к доказательству теоремы 2". По предположению индукции двучлен $x^{k}-r_{1}^{k}$ неприводим над $\mathbb{Q}$, двучлен $x^{k}-r_{2}^{k}-$ над $\mathbb{Q}\left(r_{1}\right), \ldots$, двучлен $x^{k}-r_{n-1}^{k}-$ над $\mathbb{Q}\left(r_{1}, \ldots, r_{n-2}\right)$. Последовательно избавляясь от иррациональности в знаменателе, представим каждое число из $\mathbb{Q}\left(r_{1}, \ldots, r_{n-1}\right)$ однозначно в виде суммы чисел вида

$a r_{1}^{l_{1}} \ldots r_{n-1}^{l_{n-1}}$, где $a \in \mathbb{Q}, 0 \leq l_{1}, \ldots, l_{n-1}<k$.

Пусть (9) неверно, т.е. $r_{n}^{l}$ при некотором $l \in\{1, \ldots, k-1\}$ равно сумме чисел вида (11). В этой сумме должно быть больше одного слагаемого, иначе $r_{n}^{l} /\left(r_{1}^{l_{1}} \ldots r_{n-1}^{l_{n-1}}\right)=$ $=a \in \mathbb{Q}$, что противоречит лемме 1 . Значит, какой-то из радикалов $r_{1}, \ldots, r_{n-1}$ встречается в двух слагаемых в разных степенях, пусть это $r_{n-1}$. Итак,

$$
r_{n}^{l}=A_{0}+A_{1} r_{n-1}+\ldots+A_{k-1} r_{n-1}^{k-1},
$$

где среди $A_{0}, \ldots, A_{k-1} \in \mathbb{Q}\left(r_{1}, \ldots, r_{n-2}\right)$ хотя бы два ненулевых. Самое интересное наступает в этот момент - трудность по сравнению со случаем $k=2$ возрастает еще больше. И вправду, при $k=2$ равенство (12) совсем не страшно: $\sqrt{p_{n}}=A_{0}+A_{1} \sqrt{p_{n-1}}$ без труда возводится в квадрат. Но при $k>2$ о возведении (12) в $k$-ю степень даже думать не хочется... На помощь вновь приходят минимальные многочлены. Но если для левой части минимальный многочлен найти легко, то для правой не очевидна даже алгебраичность.

Пример 7. Приведем к противоречию упомянутое выше равенство $\sqrt[5]{3}=a+b \sqrt[5]{2}$, где $a, b \in \mathbb{Q},-$ частный случай (12). По лемме $1, a \neq 0$ и $b \neq 0$. Согласно примеpy $5, \mu_{\sqrt[5]{3}}(x)=x^{5}-3$ и $\mu_{\sqrt[5]{2}}(x)=x^{5}-2$, а тогда $\mu_{a+b \sqrt[5]{2}}(x)=(x-a)^{5}-2 b^{5}$ (сделали линейную замену $x \mapsto \frac{x-a}{b}$ и умножили на $\left.b^{5}\right)$. Получаем противоречие:

$$
\begin{aligned}
x^{5}-3=(x-a)^{5}- & 2 b^{5}= \\
& =x^{5}-5 a x^{4}+\ldots \Rightarrow a=0 .
\end{aligned}
$$

Задача 10. Решите уравнение в натуральных числах: $\sqrt[5]{m}+\sqrt[5]{n}=2021$.

В общем случае путь к минимальному многочлену правой части равенства (12) лежит через сопряженные числа. Вот только что это такое, мы определили пока лишь для квадратичных иррациональностей, и в конце §2 наметили рассуждение с переходом к сопряженным.

Еще немного теории. Пусть число $\alpha \in \mathbb{C}$ алгебраично над полем $K$ и

$$
\mu_{\alpha}(x)=\left(x-\alpha_{1}\right) \ldots\left(x-\alpha_{k}\right)
$$

(согласно основной теореме алгебры любой многочлен над $\mathbb{C}$ раскладывается на линейные множители $)^{4}$. Числа $\alpha_{1}, \ldots, \alpha_{k}$ называются сопряженными $c$ а над $K$. Обозначим их сумму $\sigma(\alpha)$. По теореме Виета

$$
\begin{aligned}
\sigma(\alpha)= & \alpha_{1}+\ldots+\alpha_{k} \Leftrightarrow \\
& \Leftrightarrow \mu_{\alpha}(x)=x^{k}-\sigma(\alpha) x^{k-1}+\ldots
\end{aligned}
$$

Пример 7 показывает, что именно коэффициент $-\sigma(\alpha)$ будет играть ключевую роль.

Замечание. Числа $\alpha_{1}, \ldots, \alpha_{k}$ различны (для доказательства это не нужно): если $\mu_{\alpha}(x)=\left(x-\alpha_{j}\right)^{2} g(x), \quad$ то производная $\mu_{\alpha}^{\prime}(x)=2\left(x-\alpha_{j}\right) g(x)+\left(x-\alpha_{j}\right)^{2} g^{\prime}(x) \in K[x]$ имеет корень $\alpha_{j}$, хотя $\operatorname{deg} \mu_{\alpha}^{\prime}<\operatorname{deg} \mu_{\alpha}$.

Ввиду пункта 4) теоремы 3 все алгебраические над $K$ числа разбиваются на классы сопряженных, каждый из которых состоит из корней какого-то неприводимого над $K$ многочлена.

Пример 8. Разложим двучлен $x^{4}-2$ на неприводимые и разобьем его корни на классы сопряженных над каждым из полей $\mathbb{Q} \subset \mathbb{Q}(\sqrt{2}) \subset \mathbb{Q}(\sqrt[4]{2}) \subset \mathbb{Q}(\sqrt[4]{2}, i)$ (рис.4).

\footnotetext{
${ }_{4}^{4}$ Мы этим пользоваться не будем: в доказательстве все многочлены раскладываются явно.
} 


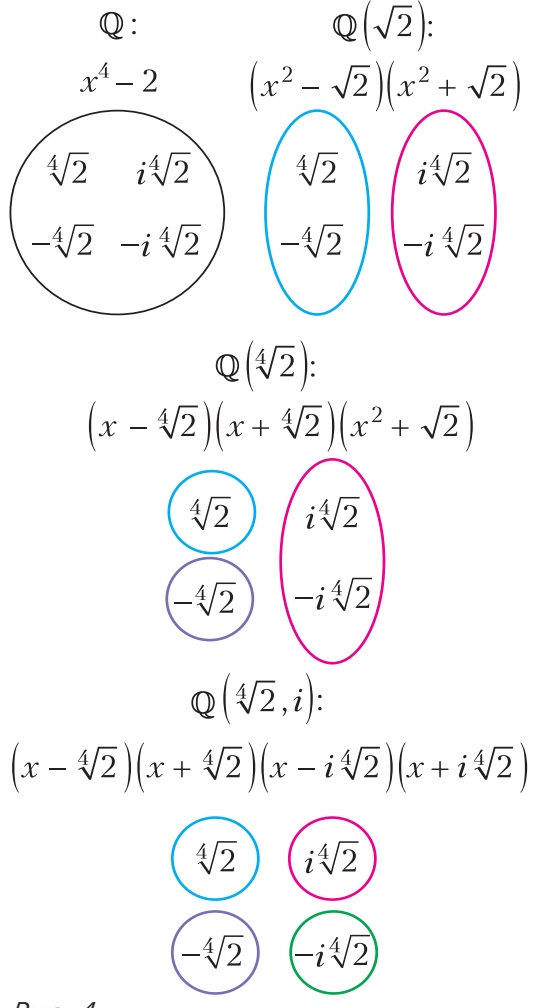

Pис. 4

Пример 9. Для квадратичных иррациональностей $a \pm b \sqrt{d} \quad(a, b, d \in K, \quad b \neq 0$, $\sqrt{d} \notin K)$ получается привычное понятие сопряженности:

$$
\begin{aligned}
\mu_{a \pm b \sqrt{d}}^{K}(x)=x^{2}-2 a x+a^{2}-d b^{2} & \\
\sigma(a \pm b \sqrt{d}) & =2 a .
\end{aligned}
$$

(Кстати, при $K=\mathbb{R}$ и $d=-1$ получаем комплексно-сопряженные числа $a \pm b i$.) Это спасает приведенное выше обоснование перехода от (3) к (4).

Пример 10. Пусть $a, b \in \mathbb{Q}, b \neq 0$. Числа $a \pm b \sqrt[k]{2}$, конечно, не будут сопряженными при $k>2$ (по аналогии с квадратичными иррациональностями). Сопряженные с числом $a+b \sqrt[k]{2}$ суть корни многочлена $\mu_{a+b \sqrt[k]{2}}(x)=(x-a)^{k}-2 b^{k}$ (аналогично примеру 7), т.е. числа $a+b \sqrt[k]{2} \varepsilon^{j}, j=0, \ldots, k-1$ (пример на рисунке 5). Знак \pm при $k=2$ объясняется тем, что $\varepsilon_{2}=-1$.

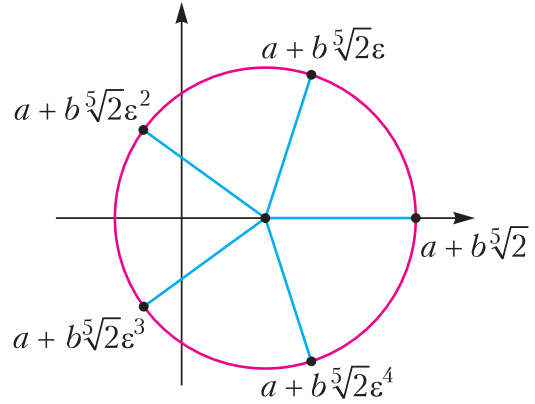

РИс. 5

Лемма 5. Пусть $K-$ подполе в $\mathbb{R}, r \in \mathbb{R}$, $r \notin K, r^{k} \in K$ для некоторого $k \in \mathbb{N}$. Тогда $\sigma(r)=0$.

Шаг 4: переход к сопряженным числам. Пример 10 подсказывает вид сопряженных к числу вида (10).

Задача 11. Опровергните равенство $\sqrt[3]{3}=a+b \sqrt[3]{2}+c \sqrt[3]{4}$, где $a, b, c \in \mathbb{Q}$. Для это2о, обозначив $f(x)=a+b x+c x^{2} u \varepsilon=\varepsilon_{3}$, покажите, что сопряженные $c$ правой частью суть

$$
\begin{gathered}
f(\sqrt[3]{2})=a+b \sqrt[3]{2}+c \sqrt[3]{4} \\
f(\sqrt[3]{2} \varepsilon)=a+b \sqrt[3]{2} \varepsilon+c \sqrt[3]{4} \varepsilon^{2} \\
f\left(\sqrt[3]{2} \varepsilon^{2}\right)=a+b \sqrt[3]{2} \varepsilon^{2}+c \sqrt[3]{4} \varepsilon
\end{gathered}
$$

Лемма 6. Пусть $\alpha_{1}=\alpha, \ldots, \alpha_{k}-$ все co- $^{-}$ пряженные с числом $\alpha$, алгебраччным над полем $K, u f \in K[x]$. Тогда число $f(\alpha)$ алгебраччно над $\mathrm{K}$ и для некоторого $d \in \mathbb{N}$ $\mu_{f(\alpha)}(x)^{d}=\left(x-f\left(\alpha_{1}\right)\right) \ldots\left(x-f\left(\alpha_{k}\right)\right)$.

Приравняем суммы корней (с кратностями) многочленов в равенстве (14):

$$
d \sigma(f(\alpha))=f\left(\alpha_{1}\right)+\ldots+f\left(\alpha_{k}\right) .
$$

Нас интересует случай $\mu_{\alpha}(x)=x^{k}-\alpha^{k}$, тогда $\alpha_{j}=\alpha \varepsilon^{j-1}, j=1, \ldots, k$. Для многочлена $f(x)=c_{0}++c_{1} x+\ldots+c_{k-1} x^{k-1}$ имеем

$$
\begin{aligned}
& f(\alpha)=c_{0}+c_{1} \alpha \quad+\ldots+c_{k-1} \alpha^{k-1}, \\
& f\left(\alpha \varepsilon=c_{0}+c_{1} \alpha \varepsilon \quad+\ldots+c_{k-1} \alpha^{k-1} \varepsilon^{k-1},\right. \\
& \cdots \\
& f\left(\alpha \varepsilon^{k-1}\right)=c_{0}+c_{1} \alpha \varepsilon^{k-1}+\ldots+c_{k-1} \alpha^{k-1} \varepsilon^{(k-1)^{2}} .
\end{aligned}
$$


Сложим числа в каждом столбике. Так как $\varepsilon^{k}=1$, то при $j=1, \ldots, k-1$

$$
1+\varepsilon^{j}+\varepsilon^{2 j}+\ldots+\varepsilon^{(k-1) j}=\frac{1-\varepsilon^{k j}}{1-\varepsilon^{j}}=0 .
$$

Значит, $f(\alpha)+f(\alpha \varepsilon)+\ldots+f\left(\alpha \varepsilon^{k-1}\right)=k c_{0}$, откуда

$$
\sigma\left(c_{0}+c_{1} \alpha+\ldots+c_{k-1} \alpha^{k-1}\right)=\frac{k}{d} \cdot c_{0} .
$$

Наконец мы готовы привести равенство (12) к противоречию. Пусть $A_{j}-$ первый ненулевой коэффициент в ряду $A_{0}, \ldots, A_{k-1}$. Разделим равенство (12) на $r_{n-1}^{j}$ :

$$
\frac{r_{n}^{l}}{r_{n-1}^{j}}=A_{j}+A_{j+1} r_{n-1}+\ldots+A_{k-1} r_{n-1}^{k-j-1} \text {. }
$$

Пусть $K=\mathbb{Q}\left(r_{1}, \ldots, r_{n-2}\right), R=r_{n}^{l} / r_{n-1}^{j}$. Тогда $R \notin K$, иначе $R=A_{j}$ и $A_{j+1}=\ldots=A_{k-1}=0$ ввиду линейной независимости $1, r_{n-1}, \ldots, r_{n-1}^{k-1}$ над $K$ (напомним, среди чисел $A_{j}, \ldots, A_{k-1}$ хотя бы два ненулевых). При этом $R^{k}=p_{n}^{l} / p_{n-1}^{j} \in \mathbb{Q}$, поэтому $\sigma(R)=0$ по лемме 5 . В то же время значение $\sigma$ от правой части (16), согласно (15), пропорционально $A_{j}$ (играющего роль $c_{0}$ ), а потому не равно 0. Полученное противоречие завершает доказательство теоремы 2.

Фактически мы доказали большее: каждое число в поле $\mathbb{Q}\left(r_{1}, \ldots, r_{n}\right)$ представляется в виде линейной комбинации $k^{n}$ чисел

$$
r_{1}^{l_{1}} \ldots r_{n}^{l_{n}} \text {, где } 0 \leq l_{1}, \ldots, l_{n}<k
$$

с однозначно определенными рациональными коэффициентами. На языке векторов, эти числа образуют базис поля $\mathbb{Q}\left(r_{1}, \ldots, r_{n}\right)$ над $\mathbb{Q}$. Такое представление достигается избавлением от иррациональности в знаменателе, а однозначность коэффициентов равносильна линейной независимости данной системы.

Короткое доказательство с помощью следа. Используя чуть больше сведений об алгебраических числах либо владея началами линейной алгебры, можно совсем коротко доказать теорему 1 (не сводя ее к теореме 2). В основе рассуждения также лежит некоторая величина, пропорциональная сумме сопряженных. Но при этом она обладает замечательным свойством линейности, благодаря чему техника сводится к минимуму.

Функция $f: L \rightarrow \mathbb{C}$, где $L-$ подполе в $\mathbb{C}$, называется линейной (точнее, $\mathbb{Q}$-линейной), если $f(a x+b y)=a f(x)+b f(y)$ для всех $x, y \in L \quad$ и $a, b \in \mathbb{Q}$. Пусть $L=$ $=\mathbb{Q}\left(\sqrt[k_{1}]{Q_{1}}, \ldots, \sqrt[k_{N}]{Q_{N}}\right)$. В $\S 4$ мы покажем, что существует линейная функция $\operatorname{tr}: L \rightarrow \mathbb{C}$, называемая следом (от англ. trace - след), которая обладает свойством:

для каждого $\alpha \in L$ найдется такое $d \in \mathbb{N}$, что $\operatorname{tr}(\alpha)=d \sigma(\alpha)(17)$

$(\sigma(\alpha)$ определено в (13)). Предположим, что $a_{1} \sqrt[k_{1}]{Q_{1}}+\ldots+a_{N} \sqrt[k_{N}]{Q_{N}}=0$, где $a_{1}, \ldots$ $\ldots, a_{N} \in \mathbb{Q}$ не все равны 0 . Будем считать, что $a_{N} \neq 0$. Неожиданным образом уединим не радикал, а коэффициент. Разделим обе части на $\sqrt[k_{N}]{Q_{N}}$ и обозначим $R_{i}=\sqrt[k_{i}]{Q_{i}} \sqrt[k_{N}]{Q_{N}}, i=1, \ldots, N-1$ :

$$
-a_{N}=a_{1} R_{1}+\ldots+a_{N-1} R_{N-1} .
$$

Так как $R_{i}^{k_{i} k_{N}} \in \mathbb{Q}$ и по условию $R_{i} \notin \mathbb{Q}$, то $\sigma\left(R_{i}\right)=0$ по лемме 5 , а тогда $\operatorname{tr}\left(R_{i}\right)=0$ в силу (17). Отсюда в силу линейности след от правой части равенства (18) равен 0. В то же время $\sigma\left(-a_{N}\right)=-a_{N} \neq 0$ и по (17) $\operatorname{tr}\left(-a_{N}\right) \neq 0$. Это противоречие завершает доказательство теоремы 2.

\section{(Продолжение следует)}

\section{Литература}

1. Э.Б.Винберг. Курс алгебры.- М.: МЦНМО, 2019.

2. Л.Камнев. Иррациональность суммы радикалов. - «Квант», 1972, №2.

3. А.Канунников. Алгебра и геометрия комплексных чисел. - «Квант», 2017, № 5,6.

4. А.Л.Канунников. Алгебраические числа как векторы. - Математическое просвещение, сер.3, вып.26, 2020, с.91-122.

5. В.Олейников. Иррациональность и неприводимость. - «Квант», 1986, № 10.

6. В.А.Уфнаровский. Математический аквариум. - М.:МЦНМО, 2010.

7. I.Richards. An Application of Galois Theory to Elementary Arithmetic. - Advances in Mathematics, 13, 1974, p. 268-273. 